\title{
Incidence and surveillance of infections from Clostridium difficile: the experience at the Galliera of Genoa in the three-year period 2004-2006
}

David Usiglio*, Anna Maria Marrapodi*, Mariangela Lanata*, Paola Sansone*, Marco Mori*, Paola Fabbri**, Nadia Cenderello**, Mauro Nelli**, Roberto Tramalloni**, Maria Paola Crisalli****, Giovanni Cassola****

*S.C. Laboratorio Analisi Chimiche Cliniche e Microbiologiche

**Controllo Infezioni Ospedaliere - Direzione Sanitaria

***S.C. Reparto Malattie Infettive

E.O. Ospedali Galliera, Genova.

Key Words: Clostridium difficile, hospital infections, community infections, monitoring, impact

Incidenza e sorveglianza delle infezioni da Clostridium difficile: l'esperienza dell'ospedale Galliera di Genova nel triennio 2004-2006

\section{SUMMARY}

\section{Introduction}

Clostridium difficile is a Gram-positive bacillus, anaerobic, sporogenous ,with oro-faecal transmission. The formation of the spores can persist in a long time, encouraging the transmission. Use of broad-spectrum antibiotics, age, alteration of intestinal flora are the most common risk factors that expose the subject to any subsequent colonization and infection. The hospital staff is the main vehicle of transmission and the probability of contracting an infection is proportional to the duration of hospitalization in patients infected with cohabitation.

\section{Objectives}

The infections surveillance of $C$. difficile is a instrument to monitor procedures for the control of hospital infections, and may be useful to highlight and point out shortcomings in the system.

\section{Materials and Methods}

The data are the results of tests for the detection of toxins on stools during the period 2004-2006, distributed quarterly to study the seasonal, divided by individual UO the hospital and later for area hospital.

\section{Results and conclusions}

The incidence of infection from $C$. difficile in the last three years has been in constant increase (from 7 to 12.5 case/ 1000 admissions). Much of increase is attributable to community infections 3 case/ 1000 admissions (2004) up to about 6 case/ 1000 admissions (2006). Substantially increasing content of hospital infections (5-6 case/I000 admissions).

\section{INTRODUZIONE}

Clostridium difficile è considerato il principale agente patogeno responsabile di diarrea in ambito ospedaliero. Solo negli Stati Uniti vengono riferiti fino a 300.000 casi annui di diarrea e/o colite da C. difficile di origine nosocomiale, mentre i dati europei sull'incidenza annuale delle infezioni sostenute da questo patogeno -ESCMID Barbut et al, 2003- (5) vanno da 0.042 a 12.09 casi ogni 1000 ricoveri e la frequenza media di test positivi per la ricerca delle tossine è pari all' $8.2 \%$.

Secondo Bartlett -2002- (8) C. difficile è responsabile del 10-20\% dei casi di diarrea, del 50-75\% dei casi di colite e di oltre il $90 \%$ dei casi di colite pseudomembranosa in associazione all'uso di antibiotici, soprattutto a largo spettro.

C. difficile può essere ritrovato nel suolo, nella sabbia, nelle feci di cani, gatti e uccelli domestici. I pazienti colonizzati o infettati, gli ambienti e le superfici contaminate degli ospedali e delle resi-

\section{Corresponding author: David Usiglio}

S.C. Laboratorio Analisi Chimiche - Cliniche e Microbiologiche E. O.

Ospedali Galliera Mura delle Cappuccine 14 - 16128 Genova, Italy

Tel : 010/5634I05 - Fax :010/5634II8 - E-mail: david.usiglio@galliera.it 
denze per anziani e lungo degenti rappresentano tuttavia i principali serbatoi di infezione.

Il personale ospedaliero rappresenta il principale veicolo di trasmissione ed è stato dimostrato che la probabilità di contrarre un'infezione da $C$. difficile è direttamente proporzionale alla durata del ricovero e alla coabitazione con pazienti infetti (4). La popolazione microbica intestinale di un adulto sano è generalmente dotata di una naturale resistenza alla colonizzazione da $C$. difficile, ma in caso di alterazione di questa flora, la resistenza diminuisce esponendo il soggetto alla colonizzazione ed eventuale successiva infezione $(12,18)$. L'uso di antibiotici a largo spettro come Penicillina, Cefalosporina, Clindamicina e ultimamente chinolonici rappresenta il più comune fattore di rischio. L'età avanzata $(>65 \mathrm{aa})$ e gravi patologie concomitanti sono fattori indipendenti che comunque contribuiscono a produrre cambiamenti della comunità microbica intestinale.

La formazione delle spore permette al $C$. difficile di persistere nell'ambiente ospedaliero per lunghi periodi favorendone la trasmissione. Quest' ultima è di tipo oro-fecale, ma solo l' $1 \%$ delle cellule batteriche allo stato vegetativo superano l'acidità dello stomaco raggiungendo l'intestino. Le spore invece essendo acido-resistenti raggiungono il piccolo intestino dove possono trasformarsi nella forma vegetativa grazie all'azione degli acidi biliari.

Maggiore è la colonizzazione, maggiore è la produzione delle due tossine $\mathrm{A}$ e $\mathrm{B}$, il principale fattore di virulenza. $(2,3,6,10,22)$. La tossina $\mathrm{A}$ è un'enterotossina con lieve attività citotossica, mentre la tossina $\mathrm{B}$ è una citotossina, ha effetto citopatico con azione patogena molto più potente della tossina $\mathrm{A}$ con la quale interagisce in maniera sinergica (8). Solo i ceppi di $C$. difficile tossinogenici sono patogeni; sono stati descritti cluster epidemici dovuti a ceppi produttori della sola tossina B (3-5\% dei ceppi) $(2,7,17,21,24)$. Di recente è stata scoperta una nuova tossina binaria, presente nel $4 \%$ dei ceppi ; non è chiaro come questa tossina contribuisca alla patogenicità di questo microrganismo (2), anche se dal 2002 in Canada (19) e negli Stati Uniti è stato registrato un aumento di casi gravi di infezioni da $C$. difficile con più alta mortalità e maggiori complicanze e recidive. Questa maggiore virulenza è stata associata a ceppi di ribotipo 027 , tossinotipo III, produttori di tossina binaria e resistenti ai fluorochinolonici. Questo patogeno è stato trovato per la prima volta nel 1988 (2) in un caso di grave colite pseudomembranosa e per molti anni è stato considerato raro e poco importante (2).

Dal 2005 numerose epidemie da $C$. difficile ribotipo 027 sono state segnalate in vari paesi, come dimostra uno studio collaborativo condotto in Europa (14) che ha permesso di individuare la cir- colazione di questo ceppo iper-virulento anche nel nostro continente. $(1,2,10,11,13,23)$.

Il periodo di incubazione tra l'ingestione del germe e la comparsa dei sintomi non è stato chiarito (20). Nella relazione tra uso di antibiotici ed insorgenza dei sintomi è stata dimostrata la necessità di un intervallo temporale da un minimo di 1 giorno a un massimo di 6-7 settimane. La diagnosi di diarrea associata a $C$. difficile dovrebbe essere sospettata in ogni paziente ospedalizzato di età $>65 \mathrm{aa}$, eventualmente febbrile, soprattutto se l'anamnesi rivela recente uso di antibiotici o altri fattori di rischio.

La diagnosi di laboratorio si basa prevalentemente sulla ricerca di entrambe le tossine direttamente nei campioni fecali mediante test rapidi immunoenzimatici (sensibilità $85-95 \%),(6,8)$.

L'esame colturale delle feci non è raccomandato routinariamente, in quanto non discrimina i ceppi tossinogenici (patogeni) da quelli non tossinogenici $(2,11,17,18)$.

La sorveglianza delle infezioni da $C$. difficile può essere adottata quale strumento di monitoraggio della corretta applicazione delle procedure per il controllo delle infezioni ospedaliere e potrebbe inoltre divenire uno strumento per l'evidenziazione e la segnalazione di eventuali lacune del sistema e quindi un utile suggeritore di idonei interventi correttivi.

\section{MATERIALI E METODI}

I dati considerati in questo studio sono rappresentati dai risultati del test per la ricerca delle tossine del $C$. difficile su feci, relativi al triennio 2004-2005-2006, distribuiti trimestralmente per studiare la stagionalità, divisi per singole U.O. di degenza, includenti le aree medica, chirurgica e unità di terapia intensiva. È stata valutata l'incidenza dei casi positivi in relazione al numero dei ricoveri nei singoli reparti di degenza, la presenza di sospetti cluster epidemici (3 o più casi nello stesso reparto entro 10 giorni). La distinzione tra infezioni ospedaliere o comunitarie è stata fatta fino al 2004, solo in base all'intervallo tra la data di ricovero e la data del test (ospedaliera se $>3$ giorni), a partire dal 2005 mediante le schede compilate dal Servizio di Igiene Ospedaliera per ogni nuovo caso di positività al test. Dalle stesse schede sono dedotti altri dati quali: la provenienza del paziente da altre strutture ospedaliere o per lungodegenti, i sintomi diarroici al momento del ricovero o l'uso di antibioticoterapia.

\section{Il Test rapido per la Ricerca delle Tossine del CD su campione fecale}

A partire dal settembre 2005 è stato adottato il test Premier Toxins A\&B distribuito dalla ditta Meridian (18). Si tratta di un test rapido immuno- 
enzimatico qualitativo per la ricerca delle tossine $A \& B$ nei campioni di feci. Il test si effettua in microtiter con pozzetti coattati con anticorpi monoclonali e policlonali specifici per le due tossine. A tali pozzetti si aggiunge il campione di feci diluito e anticorpi policlonali anti-tossina A e B coniugati con perossidasi di rafano. Se le tossine sono presenti nel campione, gli anticorpi policlonali specifici formeranno un complesso con le tossine che rimarrà presente anche dopo una serie di lavaggi. Alla fine si aggiunge ai pozzetti un substrato cromogeno (urea perossido e tetrametilbenzidina), che in presenza del complesso tossina-coniugato diventa di colore blu. Aggiungendo la soluzione acida d'arresto della reazione il colore vira al giallo. A questo punto si effettua una lettura spettrofotometrica con valore dell'assorbanza alla doppia lunghezza d'onda di 450/630 nm. Il campione è stato considerato positivo per valori superiori a 0.100 . Il test è in grado di rilevare livelli di tossina $\mathrm{A}>=\mathrm{a}$ $1,4 \mathrm{ng} / \mathrm{ml}$ di feci e livelli di tossina $\mathrm{B}>=$ a 2,4 $\mathrm{ng} / \mathrm{ml}$ di feci. La specificità è elevata $98-100 \%$, mentre la sensibilità dichiarata è pari al $82-95 \%$. Esiste quindi la possibilità di falsi negativi dovuti principalmente a: 1) errata procedura di raccolta, conservazione e/o tempo dilazionato di trasporto del campione (quest'ultimo va conservato in frigo $4{ }^{\circ} \mathrm{C}$ entro un'ora dalla raccolta, per un massimo di 72 ore); 2) limiti di rilevazione delle tossine sopraindicati. Prima del settembre 2005 si usava un test che rilevava la presenza della sola tossina A, considerato in generale meno sensibile nella rilevazione di campioni positivi con bassi livelli di tossine (borderline) e inefficace nel rilevare ceppi produttori della sola tossina $\mathrm{B}$.

\section{RISULTATI E DISCUSSIONE}

Dal 2004 è stato riscontrato un aumento annuale costante (2005: $+50 \%$. 2006: $+21 \%)$ dell'incidenza dei casi di infezione da $C$. difficile (da 7 a 12.5 casi/1000 Ricoveri),(Figura I).

Tale dato è stato confermato sia dall'aumento del numero di pazienti ospedalizzati con infezione accertata (da 119 a 215 nel 2006) sia dall'aumento delle percentuali di positività al test per la ricerca delle tossine (2004: 25\%. 2005: 32\%. 2006: 34\%), a fronte di un costante numero di ricoveri, durante il triennio in esame, di circa 17.000 l'anno.

L'incidenza delle infezioni comunitarie è sensibilmente aumentata tra il 2004 e il 2005(+50\%) per poi stabilizzarsi nel 2006 (5.4 casi/1000 ricoveri). Diversamente, l'incidenza delle infezioni ospedaliere, lievemente incrementata tra il 2004 e il 2005 $(+15 \%)$, è aumentata maggiormente nel 2006 $(+51 \%, 7.1$ casi/1000 ricoveri), (Figura II). Questo aumento è dovuto alla tipologia di pazienti "dirottati" al nostro Ospedale per necessità di interventi chirurgici più importanti.

L'età media dei pazienti con infezione da Clostridium relativi al biennio studiato è di 76 anni con una prevalenza del sesso femminile, (figure III e IV).

L'andamento stagionale delle infezioni da $C$. difficile non è mai stato uguale nei tre anni presi in esame. In particolare: nel 2004 i picchi si sono verificati nel $1^{\circ}$ e $4^{\circ}$ trimestre, nel 2005 la tendenza è apparsa addirittura opposta con un apice nel $3^{\circ}$ trimestre, mentre nel 2006, dopo un considerevole culmine nel $1^{\circ}$ trimestre, si è registrata una costante flessione dell'incidenza, (figura V e VI ). Considerando l'andamento delle infezioni totali si nota un costante incremento del numero dei casi/ 1000 ricoveri tra il $1^{\circ}$ trimestre 2004 e il $3^{\circ}$ trimestre 2005. Nel $1^{\circ}$ trimestre 2006 si registra l'incidenza più alta del triennio (19.2 casi / 1000 ricoveri), segue un costante decremento fino alla fine dell'anno. L'incidenza delle infezioni ospedaliere è rimasta costante nel triennio (media trimestrale intorno ai 56 casi/1000 ricoveri), con un solo picco considerevole nel $1^{\circ}$ trimestre 2006 (12.4 casi /1000 ricoveri). Le infezioni comunitarie sono raddoppiate nell'arco del triennio, a partire dal 2004 (media trimestrale inferiore ai 3 casi/ 1000 ricoveri) fino al 2006 (media trimestrale di circa 6 casi/ 1000 ricoveri).

L'approfondimento delle indagini sui "sospetti cluster epidemici" verificatisi nel nostro Ospedale durante il triennio in oggetto ha dato i seguenti risultati: nel 2004 si è verificato un totale di 8 sospetti cluster epidemici, nel 200522 sospetti cluster, con il picco nel $3^{\circ}$ trimestre ( 10 cluster), nel 2006 il totale è lievemente diminuito ( totale: 17 sospetti cluster ), con picco relativo al $1^{\circ}$ trimestre (10 cluster). Si rileva, quindi, un andamento trimestrale sovrapponibile tra i picchi dell'incidenza delle infezioni da $C$. difficile e il numero di sospetti cluster epidemici. Dall'elaborazione dei dati in nostro possesso sono state fatte le seguenti considerazioni: il $65 \%$ dei pazienti aveva fatto terapia antibiotica nell'arco dei due mesi precedenti al ricovero; il 76\% dei pazienti con infezione ospedaliera aveva fatto terapia antibiotica contro il 54\% dei pazienti con infezione comunitaria. Pazienti con pregressa terapia antibiotica e provenienza da RSA o da altra struttura ospedaliera: $1 ' 85 \%$ contrae una infezione ospedaliera, mentre il $69 \%$ contrae una infezione di tipo comunitario.

Pazienti con pregressa terapia antibiotica e provenienti dalla propria abitazione: Il $73 \%$ contrae una infezione ospedaliera, solo il $45 \%$ una infezione comunitaria.

Quindi, come atteso e in accordo con la letteratura (32), i dati ci confermano che sia la lungodegenza che una pregressa terapia antibiotica sono fattori che separatamente o in maggiore misura se conco- 
mitanti, predispongono all'insorgenza di infezioni da $C$. difficile soprattutto di tipo ospedaliero.

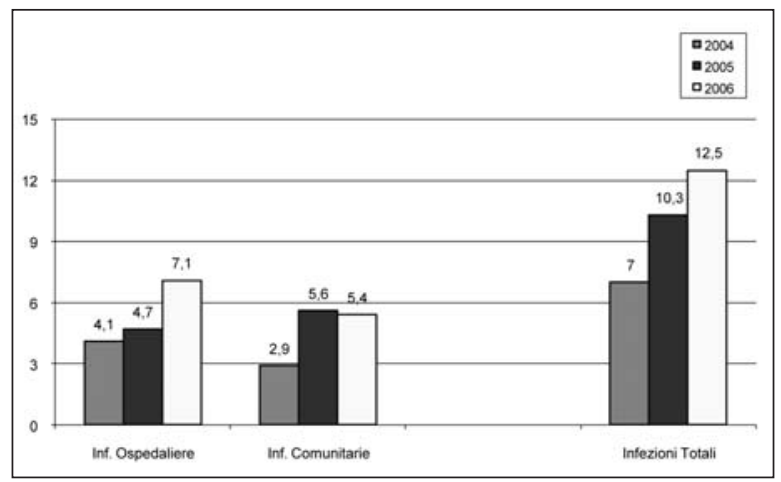

Figura I. Numero casi di infezione da Clostridium difficile/l000 ricoveri. Infezioni totali anni 2004-20052006 infezioni ospedaliere e comunitarie

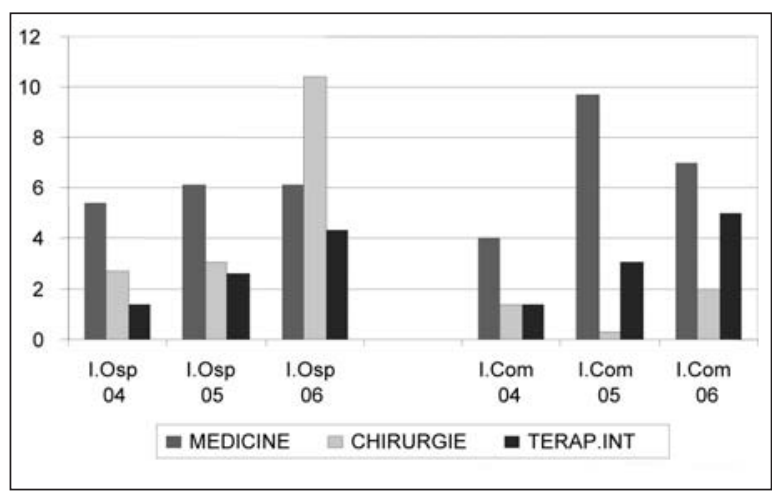

Figura II. Numero casi di infezione da CD// 000 ricoveri per aree di degenza e tipologia di infezione

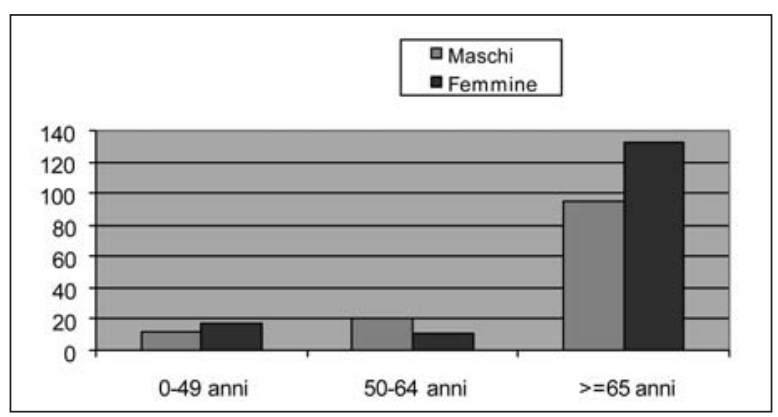

Figura III. Numero di casi di infezione da C. difficile in relazione $a$ età e sesso nel triennio 2004-2005-2006

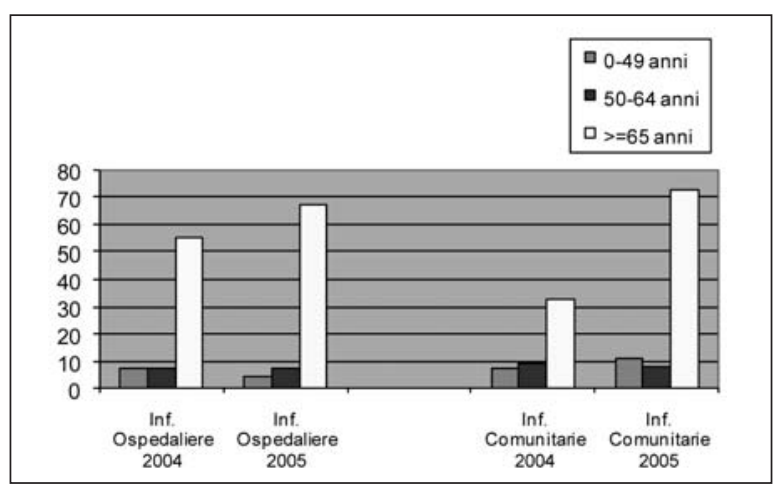

Figura IV. Numero di casi di infezioni ospedaliere/comunitarie da C. difficile in relazione all'età

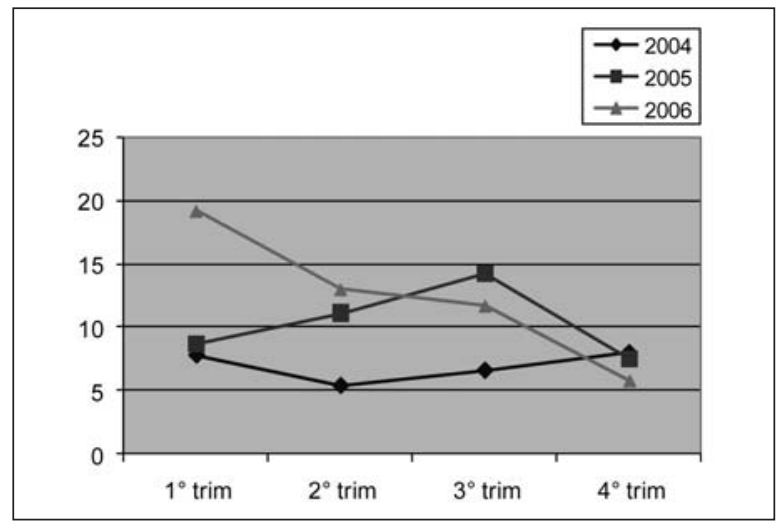

Figura V. Triennio 2004-2006: andamento stagionale dell'incidenza infezioni da C. difficile (numero di casi// 000 ricoveri)

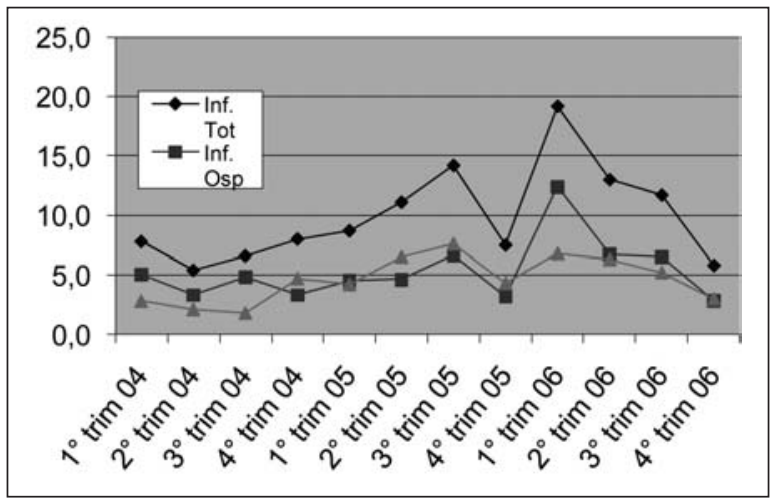

Figura VI. Incidenza trimestrale delle infezioni da C. difficile nel triennio 2004-2006. Confronto tra infezioni ospedaliere e comunitarie (numero di casil 1000 ricoveri)

\section{CONCLUSIONI}

L'andamento dell'incidenza delle infezioni da $C$. difficile nell'Ospedale Galliera relativa nel triennio in esame (2004-2006) è in costante aumento (da 7 a 12.5 casi/1000 ricoveri) confermando i dati della più recente letteratura sull'argomento (2). Tale incidenza è da considerare media, se rapportata alla variabilità dei dati pubblicati in letteratura, a seconda delle casistiche (range da 0.5 a 30 casi/1000 ricoveri ) e alla realtà del nostro ospedale: 400 posti letto con una media di circa 17.000 ricoveri l'anno in regime ordinario, età media dei pazienti elevata.

Nonostante l'aumento dei casi di infezione da $C$. difficile rilevato, il numero delle infezioni ospedaliere è stato sostanzialmente contenuto nel tempo. Una cospicua parte dell'incremento nel numero dei casi è ascrivibile soprattutto ad infezioni di tipo comunitario. Dal 2005, l'evidenza dell'aumento della casistica dell'Infezione da $C$. difficile, esterna al nostro Ospedale, è confermata sia dal cospicuo numero di infezioni comunitarie relative a pazienti provenienti da altre strutture ospedaliere e/o da lungodegenze per anziani (più di un terzo), sia dall'aumento delle percentuali di posi- 
tività al test per la ricerca delle tossine del $C$. difficile, in pazienti ambulatoriali esterni e pazienti di RSA/Spedalizzazioni territoriali.

Gli interventi attuati all'inizio del 2005 per migliorare il sistema di sorveglianza delle infezioni da $C$. difficile, si sono rivelati complessivamente efficaci: maggiore tempestività della segnalazione dei casi positivi, contenimento del numero totale delle infezioni ospedaliere (media trimestrale intorno ai 5-6 casi/1000 ricoveri), sensibilizzazione del Personale Sanitario alle problematiche della prevenzione e dell'isolamento del paziente infetto.

Purtroppo rimangono da risolvere problemi logistici, relativi ad alcuni reparti di degenza, nell'attuare le procedure di isolamento da contatto o la personalizzazione dei servizi igienici.

Nella maggioranza dei casi si tratta di difficoltà strutturali legate anche ad alcune scelte organizzative attuate nell'arco del triennio, quale l'accorpamento dei reparti di Medicina Interna e la riorganizzazione dei reparti di Chirurgia.

Nell'arco dei prossimi anni il GO-CIO (gruppo operativo comitato infezioni ospedaliere) si impegnerà a mantenere elevato il livello di attenzione della Direzione Sanitaria e Generale su queste problematiche di più difficile risoluzione. Dai nostri dati non è stato possibile dimostrare una stagionalità nell'incidenza delle infezioni da $C$. difficile. Alcuni picchi di incidenza si sono infatti verificati in periodi dell'anno diversi nell' arco del triennio studiato. Questo in parte contraddice il dato descritto in letteratura, relativo a picchi nei mesi invernali dovuti al massivo uso di antibiotici in pazienti di età superiore ai 65 anni. (16). Il dato sul numero dei sospetti cluster epidemici correla con l'andamento degli apici d'incidenza dell'infezione nei reparti di degenza. Ciò evidenzia quanto la sorveglianza sull'attuazione delle procedure comportamentali e di isolamento del paziente abbiano permesso di contenere efficacemente il numero delle infezioni ospedaliere, nonostante il trend generale in aumento.

L'attenzione sull'appropriatezza della richiesta e l'idoneità del campione, la migliore sensibilità diagnostica del nuovo test per la ricerca delle tossine e la diminuzione dei tempi di refertazione hanno evidenziato quanto può essere incisiva l'azione del Laboratorio di Batteriologia nell'ottimizzazione della procedura di sorveglianza e controllo dell'infezione da Clostridiun difficile. Dal 2006 è stata conseguita una riduzione dei costi e un migliore utilizzo del tempo lavoro, evitando l'esecuzione di esami di scarsa significatività (circa un terzo, nella nostra esperienza).

Per quanto riguarda i ceppi produttori della sola tossina $\mathrm{B}$, pur non avendo sufficienti elementi che dimostrino un aumento della loro circolazione nella nostra area geografica, va tenuto presente il lieve aumento di campioni positivi registrato dal momento in cui si è iniziato ad usare il nuovo test $(+3 \%$, tra il 2005 e il 2006).

La riduzione dei tempi di refertazione e l'invio automatico (via e-mail) al Servizio di Sorveglianza Infezioni Ospedaliere, ha consentito l'attuazione di un programma di prevenzione e di sorveglianza sulla trasmissione dell'infezione più tempestivo, soprattutto nei reparti in cui si sono verificati più casi in un breve lasso di tempo (Sospetti Cluster Epidemici).

L'aumento dei casi riscontrato nel 2006 nei reparti di Chirurgia ci spinge a sorvegliare in modo particolare sia la profilassi chirurgica che la Terapia Antibiotica.

L'elevata media dell'età dei nostri pazienti (76 anni) e la dimostrazione che la maggior parte delle infezioni da Clostridium difficile riguarda pazienti di età superiore ai 65 anni, nonché, il continuo scambio di pazienti con altri ospedali e strutture residenziali per anziani, ci stimola a cercare un nuovo e maggiore confronto, reciprocamente formativo con gli operatori sanitari anche di altre strutture ospedaliere e di lungo degenza per anziani, a noi collegate.

Il fine è di limitare sia l'aumento generalizzato delle infezioni da $C$. difficile nel nostro bacino di utenza, sia l'aumento dei casi nel nostro Ospedale, relativo a pazienti con più fattori predisponenti all'infezione o già sintomatici all'ammissione in ospedale.

A questo scopo nel 2006 è stato organizzato un incontro formativo di tre ore aperto a tutto il personale sanitario sia dell'Ospedale Galliera che esterno (Medici, Infermieri, Responsabili di RSA) per illustrare tutte le problematiche relative all'infezione da Clostridium difficile, in particolare per quanto concerne le procedure di prevenzione, e isolamento del paziente infetto, la disinfezione degli ambienti, compreso gli aspetti terapeutici.

Siamo fiduciosi che la sorveglianza specifica di alcuni reparti a rischio (adesione stretta alle procedure di prevenzione e controllo dell'infezione) e la vigilanza sulle più comuni cause predisponenti (fattori di rischio), ci consentiranno di realizzare l'obiettivo di contenere il numero di infezioni ospedaliere da $C$. difficile, nonostante il costante aumento dell'incidenza e la paventata circolazione di ceppi più virulenti $(14,15)$.

Consapevoli che il limite della nostra indagine vada necessariamente ricondotto alla realtà e alle peculiarità del nostro Ospedale, auspichiamo uno studio più approfondito su alcune delle criticità emerse. In questo senso sarebbe rilevante la condivisione con altri ospedali a livello regionale e nazionale dei dati relativi alle infezioni da $C$. difficile. 


\section{BIBLIOGRAFIA}

1. Aa.Vv. Clostridium difficile : findings and recommendations from a review of the epidemiology and a survey of directors of infection prevention and control in England. Health Protection Agency (July 24, 2006); 1-55

2. Aa.Vv. Emergence of Clostridium difficile-associated disease in Canada, the United States of America and Europe. CDC, Atlanta, George, USA, March 3 2006: $1-21$

3. Åkerlund Thomas et al. Correlation of disease severity with fecal toxin levels in patients with Clostridium difficile-Associated diarrhea and distribution of PCR ribotypes and toxin yelds in vitro of corresponding isolates. JCM, Feb 2006; $44 \mathrm{n}^{\circ} 2: 353-358$

4. Barbut $\mathrm{F}$ et al. Epidemiology of Clostridium difficileassociated infections. Clin Microbiol Infect, 2001; 7: 405-410

5. F.Barbut, M. Delmée, J.S. Branzier, et al and the ESCMID Study Group on Clostridium difficile ESGCD. A European survey of diagnostic methods and testing protocols for Clostridium difficile. European Society of Clinical Microbiology and Infectious Disease, CMI, 2003; 9, 989-996

6. Bartlett J.G. Antibiotic Associated Diarrhea. N.Engl.J.Med.. Vol.346 : 334-339. Jan 31, 2002

7. Bélanger SD et al. Rapid detection of Clostridium difficile in feces by real time PCR. J Clin Microbiol, 2003; 41: 730-734

8. Delmée M. Laboratory diagnosis of Clostridium difficile disease. Clin Microbiol Infect, 2001; 7: 411-416

9. Donskey Curtis J.. The role of the Intestinal tract as a reservoir and source for transmission of Nosocomial Pathogens. CID 2004:39 (15 July) 219-225

10. Gonçalves $\mathrm{C}$ et al. Prevalence and characterization of binary toxin (actin-specific ADP-ribosyltransferase) from Clostridium difficile. J Clin Microbiol, 2004; 42: 1933-1939

11. Health Protection Agency. Management, Prevention and Surveillance of Clostridium difficile. Interim find- ings from a National Survey of NHS acute trusts in England. December 2005

12. Johnson S, Gerding DN Clostridium difficile-associated diarrhea. Clin Infec Dis, 1998; 26: 1027-1036

13. Kato $\mathrm{H}$ et al. Identification of toxin A-negative, toxin B-positive Clostridium difficile by PCR. J Clin Microbiol, 1998; 36: 2178-2182

14. Kuijper EJ, Coignard B, Brazier J, et al. Update of Clostridium difficile-associated disease due to PCR ribotype 027 in Europe. Eurosurveillance monthly releases, June 2007; vol 12 n 6

15. Kuijper EJ, van den Berg RJ, Debast S, et al. Clostridium difficile ribotype 027 , toxinotype III, the Netherlands. Emerging Infectious Diseases, May 2006; vol $12 n^{\circ}$ 5: 827-83

16. Lennox K. et al. Secular Trends in Hospital-Acquired Clostridium difficile Disease in the United States, 1987-2001. JID $2004 ; 189$ :1585-1589

17. National Clostridium difficile Standards Group. Report to the Department of Health February 2003. England

18. O'Connor D et al. Evaluation of methods for detection of toxins in specimens of feces submitted for diagnosis of Clostridium difficile-associated diarrhea. J Clin Microbiol, 2001; 39: 2846-2849

19. Pepin J. et al. Mortality attributable to nosocomial Clostridium difficile associated disease during an epidemic caused by hypervirulent strain in Quebec. CMAJ Oct. 25,2005; 173(9)

20. Rupnik M. How to detect Clostridium difficile variant strains in a routine laboratory. Clin Microbiol Infect, 2001; 7: 417-420

21. Voth DE et al. Clostridium difficile toxin : mechanism of action and role in disease. Clin Microbiol Rev 2005; 18: 247-263

22. Warny M, Pepin J, Fang A, et al. Toxin production by an emerging strain of Clostridium difficile associated with outbreaks of severe disease in North America and Europe. Lancet (2005) 366, 1079-84

23. Wilkins Tracy D., David M. Lyerly. Clostridium difficile Testing: after 20 years, still challenging. J.Clin.Microbiol., Feb. 2003, p. 531-534 